\title{
DYNAMIC LOADING FACTORS OF INDIVIDUAL JOGGING FORCES
}

\author{
Vitomir Racic, James M.W. Brownjohn, Aleksandar Pavic \\ ${ }^{1}$ University of Sheffield \\ Department of Civil and Structural Engineering \\ Sir Frederick Mappin Building \\ Mappin Street, Sheffield, S1 3JD \\ e-mails: v.racic@sheffield.ac.uk \\ a.pavic@sheffield.ac.uk \\ james.brownjohn@sheffield.ac.uk
}

Keywords: human-induced vibrations, footbridges, running, dynamic loading.

\begin{abstract}
With increasingly popular marathon events in urban environments, such as in London and New York City, structural designers are faced with a great deal of uncertainty when assessing dynamic performance of bridges occupied and dynamically excited by people running. While the dynamic loads induced by people walking have been intensively studied since the infamous lateral sway of the London Millennium Bridge in 2000, reliable and practical descriptions of running excitation are still very rare and limited.
\end{abstract}

This paper makes a step forward by bringing together a unique database of individual jogging force records and their simple mathematical model which can be used in everyday design practice. The forcing data has been collected in Vibration Engineering Section Laboratory in the University of Sheffield using a state-of-the-art instrumented treadmill, which is commonly used in clinical studies of human gait and sports biomechanics. The modelling strategy featuring Fourier harmonics of measured jogging force-time histories is adopted from a popular design guidelines for human walking excitation of structures.

The results show a great scatter in the DLF data and no strong link with jogging footfall rate, which is the case with walking forces. This clearly suggests that traditional deterministic Fourier based approach is not the best modelling strategy for jogging loading. Uncertainty and inter-personal randomness of the force amplitudes indicate that stochastic - rather than deterministic models of jogging forces should provide more reliable predictions of the bridge dynamics. These forces could be modeled in a similar fashion as other key dynamic loading of structures characterized by great randomness and uncertainty, such as wind and earthquake. 


\section{INTRODUCTION}

In the last fifteen years substantial developments in workmanship and structural materials have enabled bridge engineers to promote much more slender designs than ever. As a result, modern bridges are at a considerable risk of developing resonance when occupied and dynamically excited by people moving during ordinary activities, such as walking, jumping and running [1]. Excessive dynamic responses recorded worldwide on at least a dozen bridges of different structural forms and sizes under groups and crowds of active people clearly prove the point [2].

While the loads induced by walking [3-7] and jumping [8-9] have been intensively studied, reliable and practical descriptions of jogging forces are still very rare and limited [10-12]. In the context of this paper, jogging refers to running at a slow or leisurely pace, usually slower than $10 \mathrm{~km} / \mathrm{h}$. Jogging is common in urban environments, hence brings a potential hazard to vibration serviceability performance of bridge structures.

Much research into jogging forces has been in the domain of biomechanics as the contact forces between the feet and supporting ground (thus generally called 'ground reaction forces' - GRFs) provide useful diagnostics for medical and sports applications [13]. Medics introduced instrumented force measuring treadmills which can be used to carry out even highspeed running studies in very small laboratories [14]. However, no database of jogging forces in the form of continuously recorded signals has yet been found that can be used for development of their statistically reliable analytical model for application in the civil engineering context.

The lack of fundamental forcing data and their reliable mathematical models has resulted in a total lack of formal design guidance regarding jogging/running excitation of bridges. The present study aims to change this situation by bringing together: (1) a comprehensive database of continuously measured vertical jogging loads generated by individuals on flat stationary surfaces, and (2) a simple mathematical characterisation of the measurements motivated by the Fourier-based modelling approach adapted from a popular design guideline for walking loading [15]. The models could be applied to cases of incipient instability, i.e. when vertical bridge motion is still not significantly perceptible to the jogger, and is ideally suited for considering cases of multiple joggers in a group or crowd.

\section{EXPERIMENTAL DATA COLLECTION}

The tests were carried out in the Vibration Engineering Section Laboratory in the University of Sheffield (UK). Continuously measured individual force records were collected on a state-of-the-art instrumented treadmill ADAL3D-F (Figure 1).

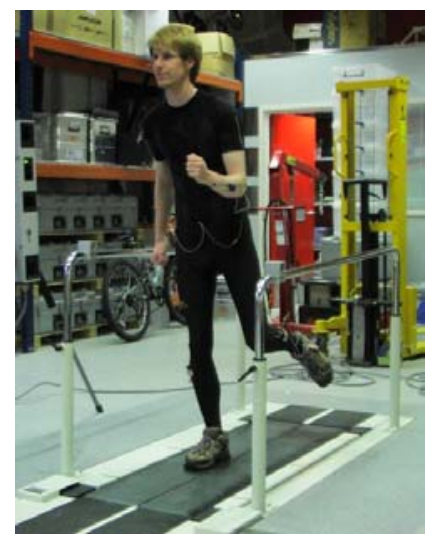

Figure 1: Experimental setup. 
Speed of the belt rotation (here also called 'treadmill speed') can be controlled and monitored remotely either with a control panel or with bespoke software, run from the data acquisition PC. Similar to fitness treadmills, the remote control panel and the treadmill itself are equipped with a safety stop switch. Rotational speed of ADAL3D-F belts is in the range 0.1$10 \mathrm{~km} / \mathrm{h}$.

All participants wore comfortable running footwear. Those who had no experience with treadmill jogging were given a brief training prior to the force collection supervised by a qualified instructor. During each individual test, participants were asked to jog on the treadmill at a fixed speed. Pacing rate was not prompted by any stimuli such as a metronome, and it was determined only from subsequent analysis of the generated force signals (see Section 3). Each test was completed when at least 64 successive footfalls were recorded. Rests were allowed between successive tests.

Acquisition of the force records started at the slowest self-selected speed at which jogging, rather than walking, is more comfortable. In $95 \%$ of test subjects processed, this speed was $6.5 \mathrm{~km} / \mathrm{h}$. In each following test the running speed was increased by $0.5 \mathrm{~km} / \mathrm{h}$, until it added up to $10 \mathrm{~km} / \mathrm{h}$.

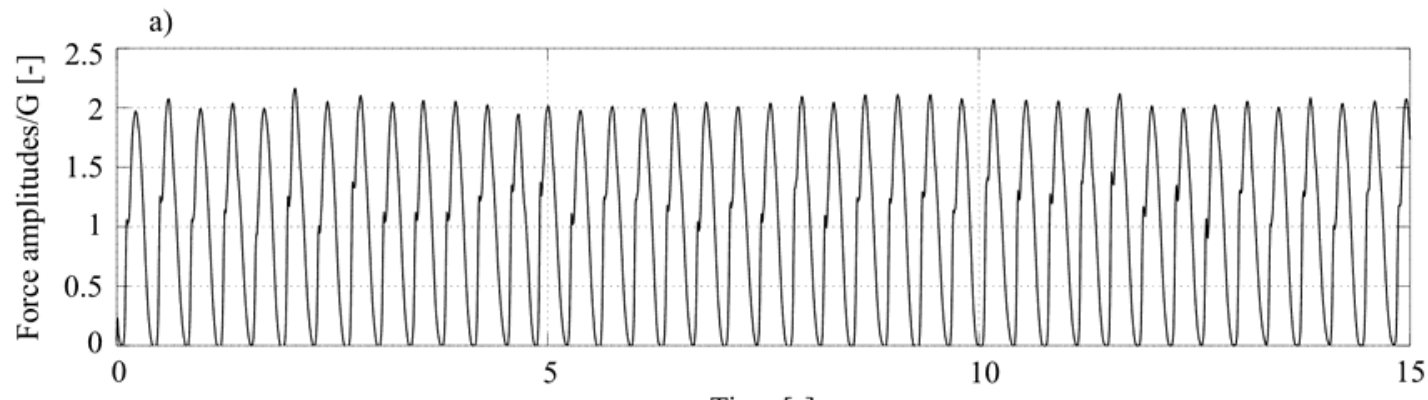

Time $[\mathrm{s}]$

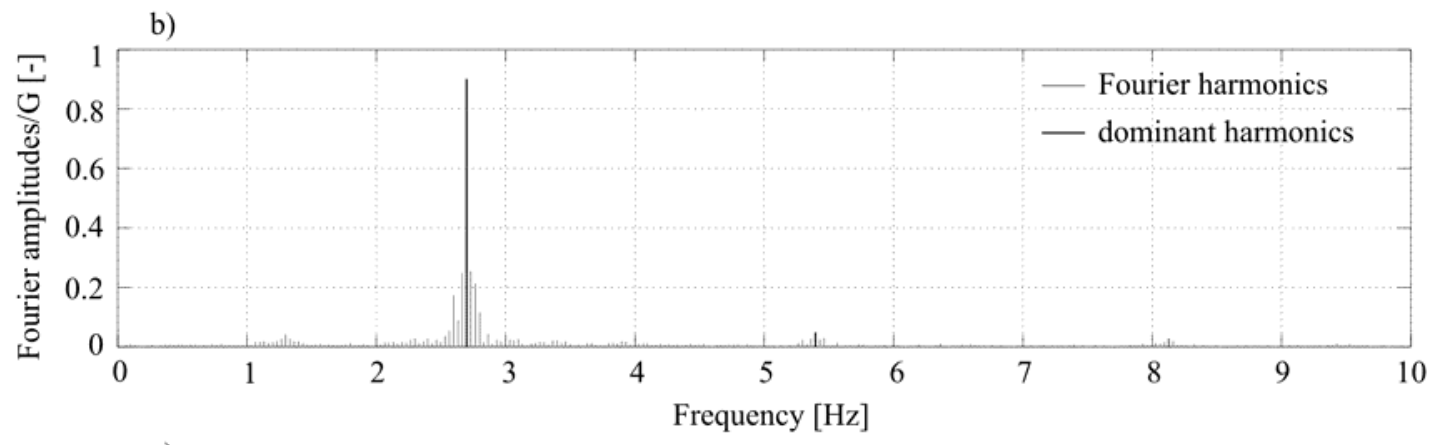

c)

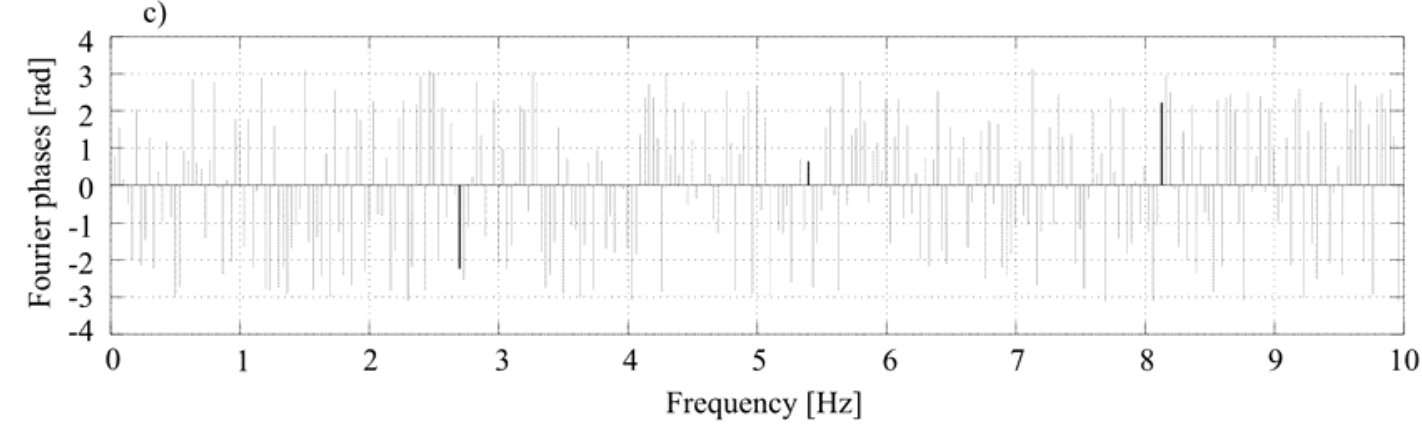

Figure 2: An example of measured force signal: (a) force-time history and its Fourier (b) amplitude and (c) phase spectra calculated from 64 successive footfalls.

In total, 24 volunteers (18 males and 6 females, body mass $68.3 \pm 12.2 \mathrm{~kg}$, height $167.2 \pm 7.1$ $\mathrm{cm}$, age $24.2 \pm 7.6$ years) were drawn from students, academics and technical staff of the Uni- 
versity of Sheffield. All together they generated 181 vertical jogging force-time histories of kind illustrated in Figure 2. In the remaining part of the paper, this unique database is used to provide their mathematical representation based on the Fourier series.

\section{DATA ANALYSIS AND MODELLING}

Measured force-time histories of human-induced loading are invariably near-periodic [1], indicating their narrow band nature (Figure 2b). However, to simplify their mathematical representations for design purposes, they are usually assumed perfectly periodic, deterministic and presentable via Fourier series [15]:

$$
F(t)=G \sum_{j=1}^{m} \alpha_{i} \sin \left(2 \pi f_{r} t-\varphi_{i}\right)
$$

Here $F(t)$ represents the total force at time $t$, with $G$ representing the body weight in the same unit. A total of $\mathrm{m}$ harmonics are considered, capturing peaks $\alpha_{\mathrm{i}}$ from the Fourier amplitude spectrum (Figure 2) at integer multiples of the footfall rate $f_{j}$. In the literature, $\alpha_{i}$ coefficients are commonly known as 'dynamic load(ing) factors' (DLFs). On the other hand, values of the phase angles $\varphi$ i have never been publicised in detail and are usually ignored in Equation (1).

Fitting Equation (1) to the numerous database of jogging force records established in the previous section can provide a valuable insight into their inter-subject variability. Shapes of the histograms shown in Figure3a suggest that jogging footfall rate is not a normally distributed as in case of walking [7,16-18]. The normal probability plots in Figure $3 \mathrm{~b}$ further proves the point. Moreover, fitting a wide range of other probability density functions, such as the family of skewed distributions [19], showed a lack of statistical significance.
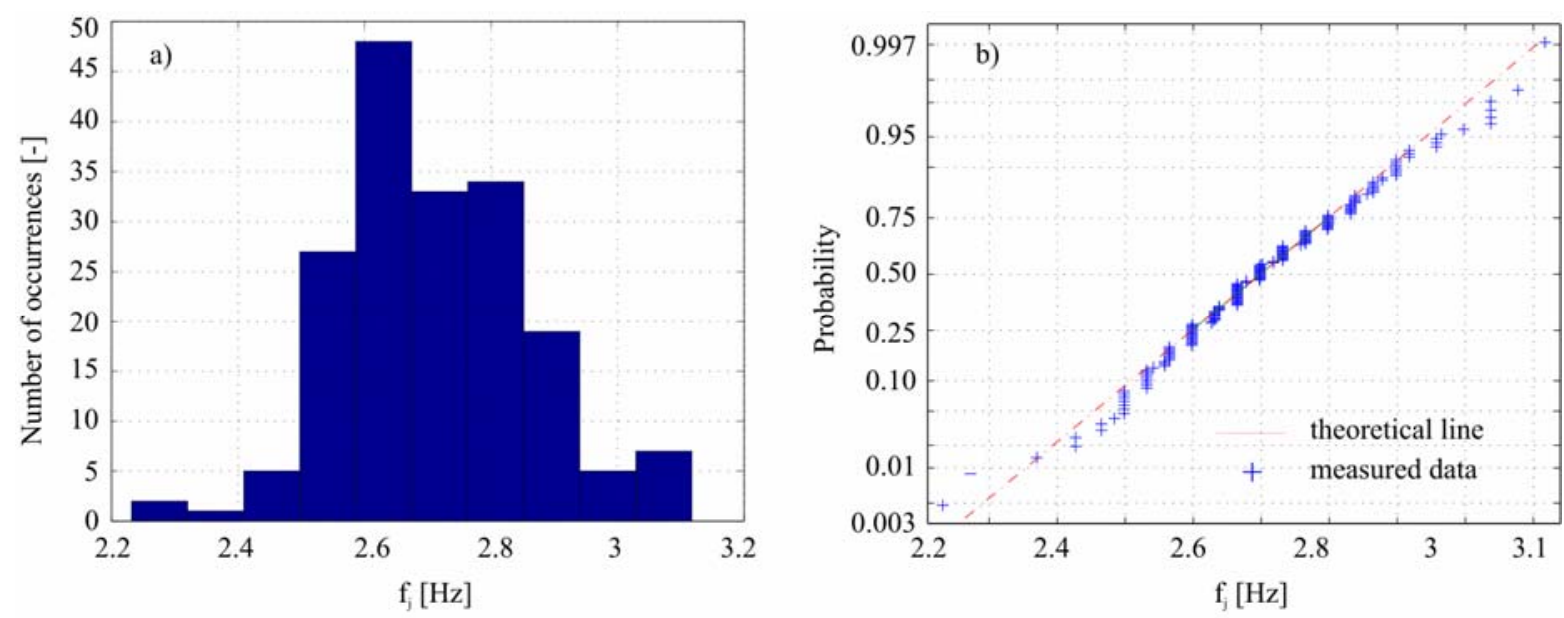

Figure 3: (a) Histogram of footfall rates and (b) the corresponding normal probability plot.

Apart from the linear trend between jogging speed and footfall rate $(\rho=0.39)$, Figure 4 illustrates a large scatter of the running frequencies at fixed running speeds, and vice versa. This observation can play a key role in simulating reliably jogging excitation of bridges due to dense crowd situations (e.g. marathon events) where jogging speed of individuals in a crowd can be controled by spatial constrains and the crowd flow.

Graphs shown in Figure 5 indicate that jogging force amplitudes generally increase as people increase their footfall rate. Due to the apparently large scatter of the DLF values at a fixed 
running frequency, their relationship cannot be described reliably as a deterministic function, such as the linear and polynomial curve fits suggested for vertical walking loads elsewhere $[15,20]$. Moreover, histograms of DLFs corresponding to a narrow range $0.1 \mathrm{~Hz}$ around the most frequent footfall rate in the sample $\mathrm{f}_{\mathrm{j}}=2.7 \mathrm{~Hz}$ do not resemble any known probability density function (Figure 6), which is a key condition for probabilistic curve fitting [19]. Also, there is no strong link between the fundamental DLF1 and $\mathrm{DLF}_{\mathrm{i}}(\mathrm{i} \geq 2)$ of higher harmonics, as shown in Figure 7.

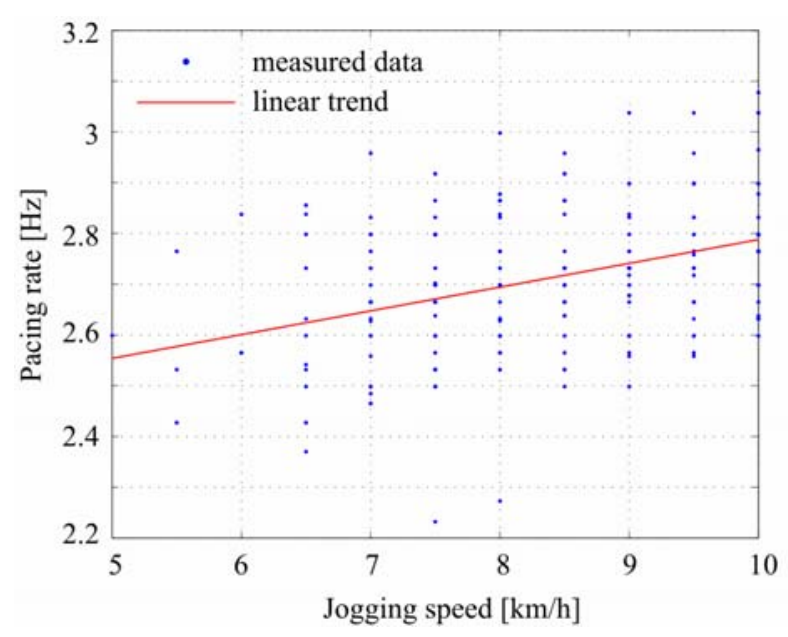

Figure 4: Jogging speed vs. footfall rate.
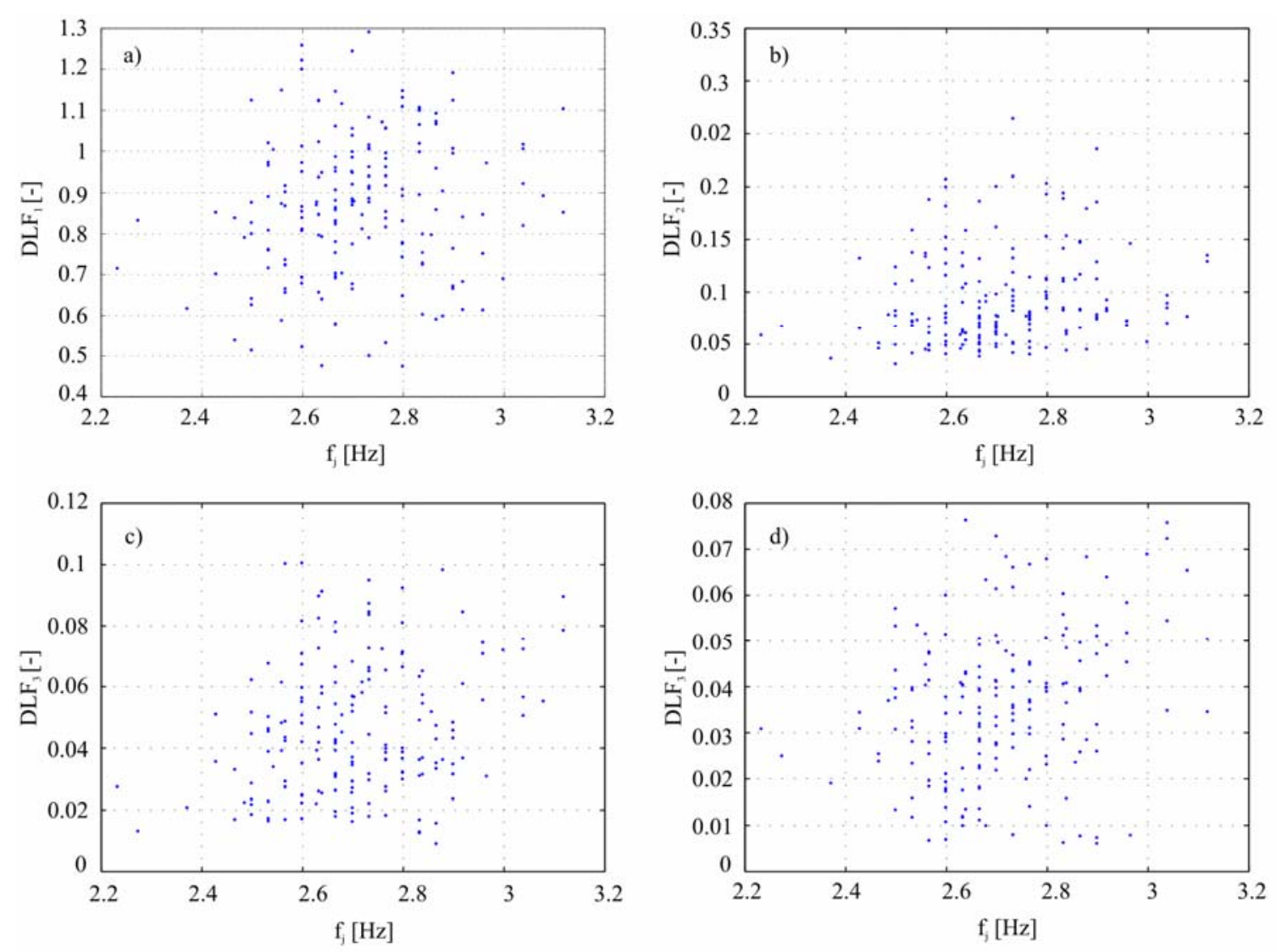

Figure 5: DLFs of the first four dominant harmonics vs. footfall rate. 

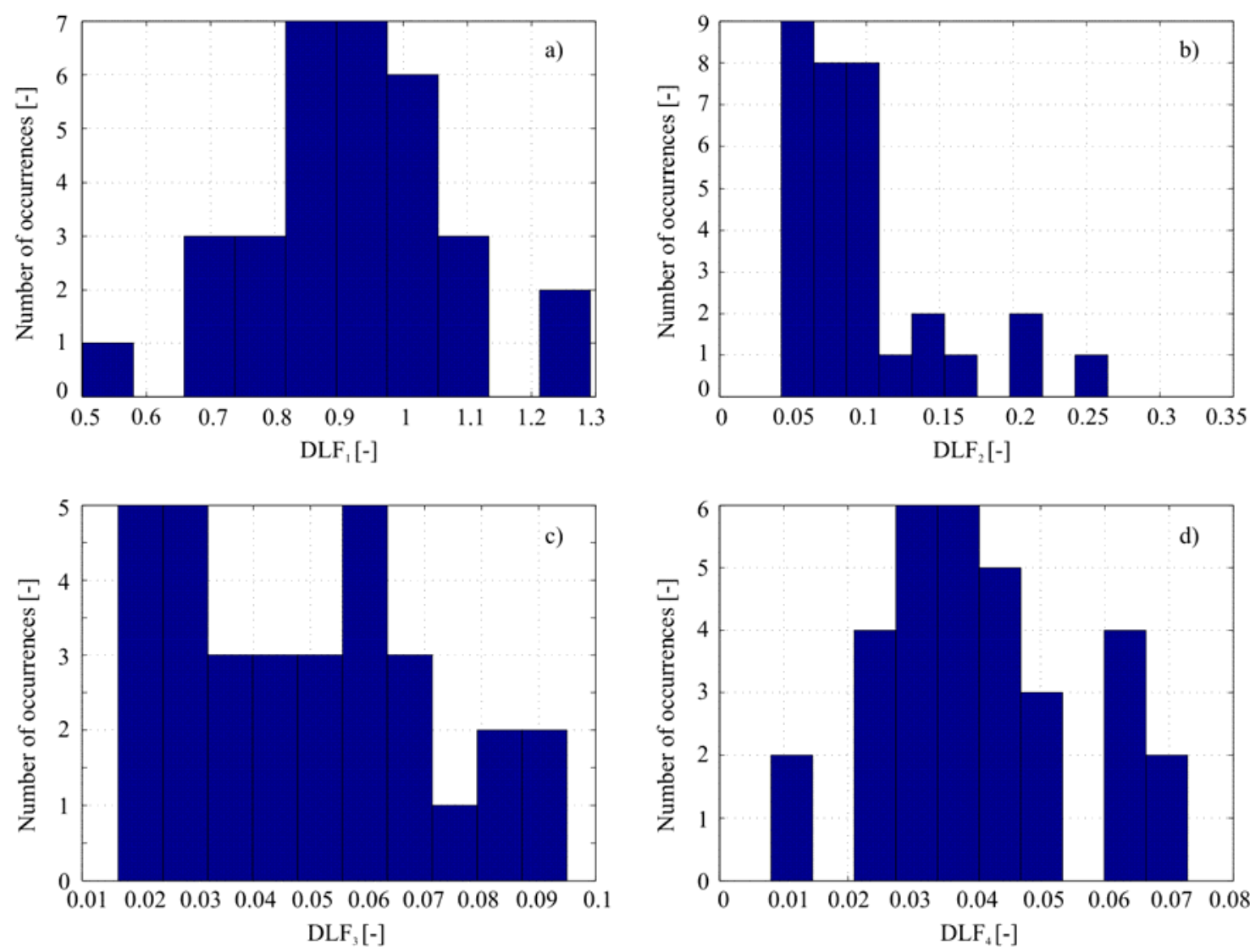

Figure 6: Histograms of DLFs corresponding to the most frequent footfall rate $\mathrm{fj}=2.7 \mathrm{~Hz}$.
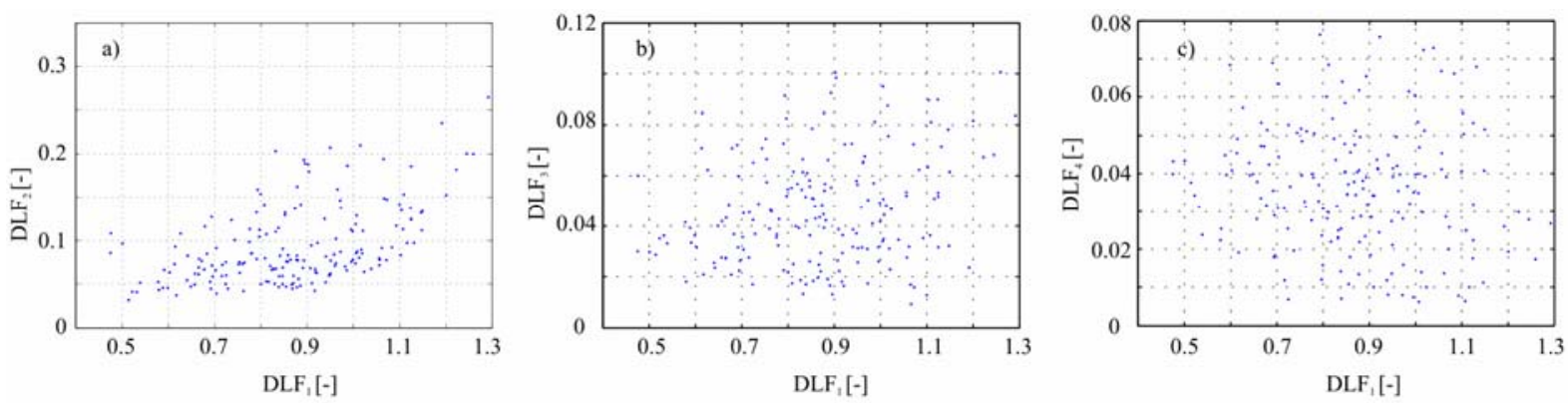

Figure 7: Fundamental DLF1 vs. (a) $\mathrm{DLF}_{2}$, (b) $\mathrm{DLF}_{3}$ and (c)DLF 4.

\begin{tabular}{ccccc}
\hline $\mathrm{f}_{\mathrm{j}}[\mathrm{Hz}]$ & $\mathrm{DLF}_{1}[-]$ & $\mathrm{DLF}_{2}[-]$ & $\mathrm{DLF}_{3}[-]$ & $\mathrm{DLF}_{4}[-]$ \\
\hline$<2.5$ & {$[0.514,1.125]$} & {$[0.031,0.132]$} & {$[0.013,0.062]$} & {$[0.013,0.057]$} \\
{$[2.5,2.6)$} & {$[0.476,1.245]$} & {$[0.039,0.200]$} & {$[0.016,0.091]$} & {$[0.010,0.076]$} \\
{$[2.6,2.7)$} & {$[0.475,1.292]$} & {$[0.040,0.264]$} & {$[0.018,0.095]$} & {$[0.008,0.068]$} \\
{$[2.7,2.8)$} & {$[0.591,1.191]$} & {$[0.044,0.236]$} & {$[0.009,0.098]$} & {$[0.006,0.068]$} \\
{$[2.8,2.9)$} & {$[0.613,0.971]$} & {$[0.052,0.146]$} & {$[0.031,0.085]$} & {$[0.008,0.069]$} \\
{$[2.9,3)$} & {$[0.820,1.012]$} & {$[0.070,0.097]$} & {$[0.051,0.076]$} & {$[0.035,0.076]$} \\
$\geq 3$ & {$[0.820,1.104]$} & {$[0.070,0.135]$} & {$[0.051,0.090]$} & {$[0.035,0.076]$} \\
\hline
\end{tabular}

Table 1: Lower and upper bands of DLF values for the given range of jogging rate $f_{j}$.

All these results have demonstrated large inter-subject randomness of jogging forces which cannot be modelled reliably using the popular deterministic Fourier series approach. There- 
fore, this study suggests randomization of DLF values in Equation (1). For the given footfall rate, DLF amplitudes are calculated randomly and equally likely using parameters of uniform distributions summarised in Table 1.

\section{DISCUSSIONS AND CONCLUSIONS}

The lack of fundamental forcing data and their reliable mathematical characterisation are the key reasons for a total lack of a formal design guidance regarding jogging excitation of bridges. To change this situation, this interdisciplinary study first established a unique database comprising 181 individual force signals collected from 24 individuals running on a biomedical instrumented treadmill at a wide range of speeds. The measurements are then fitted to the Fourier series model transferred and adopted from a popular contemporary design guideline on dynamic loads due to pedestrians walking. However, deterministic modelling approach was shown to be unreliable since the extracted force parameters are characterised by significant randomness. Therefore, the force parameters are described here using simple statistics.

Being derived from the force data recorded on a stiff ground (i.e. treadmill), the current version of the model represents only jogging excitation of structures which do not vibrate perceptibly, i.e. cases when they are not significantly affected by the structural vibrations. However, perceptible motion of the structure is just one type of cue or stimulus among several others affecting human-induced loads, such as different auditory, visual and tactile stimuli. To include all these parameters in the model, DLFs and footfall rate should be modelled as a function of bridge dynamic response, human perception to vertical vibrations and different external stimuli. To the best knowledge of the authors, experimental data necessary to accomplish this challenge is currently not available anywhere in the world.

\section{REFERENCES}

[1] V. Racic, A. Pavic, J.M.W. Brownjohn, Experimental identification and analytical modelling of human walking forces: Literature review. Journal of Sound and Vibration, 326, 1-49, 2009.

[2] S. Zivanovic, A. Pavic, P. Reynolds, Vibration serviceability of footbridges under human-induced excitation: a literature review. Journal of Sound and Vibration, 279, 1-74, 2005 .

[3] S. Zivanovic, A. Pavic, P. Reynolds, Human-structure dynamic interaction in footbridges. Bridge Engineering 158 (BE4), 165-177, 2005.

[4] F. Venuti, L. Bruno, The synchronous lateral excitation phenomenon: modelling framework and an application. Comptes Rendus Mecanique, 335, 739-745, 2007.

[5] J.H.G. Macdonald, Lateral excitation of bridges by balancing pedestrians. Proceedings of the Royal Society A, 16 December 2008.

[6] M. Kasperski, C. Sahnaci, Serviceability of pedestrian structures exposed to vibrations during marathon events. In: Proceedings of IMAC XXVI, Orlando, Florida, USA, 4-7 February, 2008.

[7] E.T. Ingólfsson, C.T. Georgakis, F. Ricciardelli, J. Jönsson, Experimental identification of pedestrian-induced lateral forces on footbridges. Journal of Sound and Vibration 330(6), 1265-1284, 2011. 
[8] E. Caetano, A. Cunha, C. Moutinho, Vandal loads and induced vibrations on a footbridge. ASCE Journal of Bridge Engineering, 16(3), 375-382, 2011.

[9] V. Racic, A. Pavic, Stochastic approach to modelling near-periodic jumping force signals. Mechanical Systems and Signal Processing, 24, 3037-3059, 2010.

[10] Galbraith F.W., Barton M.V. (1970) Ground loading from footsteps. Journal of Acoustic Society of America 48 (5), 1288-1292.

[11] J.H. Rainer, G. Pernica, D.E. Allen, Dynamic loading and response of footbridges. Canadian Journal of Civil Engineering 15 (1988) 66-71.

[12] C. Sahnaci, M. Kasperski, Excitation of buildings and pedestrian structures from walking and running. In: Proceedings of EVACES07, Porto, Portugal, 24-26 October, 2007.

[13] K. Sabri, M.E. Badaoui, F. Guillet, A. Belli, G. Millet, J.B. Morin, Cyclostationary modeling of ground reaction force signals. Signal Processing, 90(4), 1146-1152, 2010.

[14] A. Belli, P. Bui, A. Berger, A. Geyssant, J.R. Lacour, A treadmill ergometer for threedimensional ground reaction forces measurement during walking. Journal of Biomechanics, 34(1), 105-112, 2001.

[15] A. Pavic, M. Willford, Vibration serviceability of post-tensioned concrete floors, Appendix G. In Post-tensioned concrete floors design handbook, technical report 43, 99$107,2005$.

[16] Y. Matsumoto, T. Nishioka, H. Shiojiri, K. Matsuzaki, Dynamic design of footbridges. In: Proceedings of IABSE, No. P-17/78, 1-15, 1978.

[17] S. Zivanovic, A. Pavic, P. Reynolds, Probability-based prediction of multi-mode vibration response to walking excitation. Engineering Structures, 29(6), 942-954, 2007.

[18] V. Racic, J.M.W. Brownjohn, Stochastic model of near-periodic vertical loads due to humans walking. Advanced Engineering Informatics, 25(2), 259-275, 2011.

[19] C. M. Bishop, Pattern recognition and machine learning. Springer, New York, USA, 2006.

[20] P. Young, Improved floor vibration prediction methodologies. ARUP Vibration Seminar, 4 October, London, UK, 2001. 